\title{
Diagnóstico e Manejo da Ansiedade Odontológica pelos Cirurgiões-Dentistas
}

\author{
Rodrigo Dutra Murrer* \\ Simone Scandiuzzi Francisco \\ Centro Universitário Leão Sampaio, Juazeiro do Norte, CE, Brasil
}

\begin{abstract}
RESUMO
O trabalho verifica o conhecimento e a prática profissional relacionados às formas de diagnóstico e manejo da ansiedade odontológica pelos cirurgiões-dentistas. Os profissionais preencheram um questionário autoaplicável sobre diagnóstico e tratamento da ansiedade odontológica. No total, 967 cirurgiões-dentistas responderam ao questionário, dos quais aproximadamente $65 \%$ relataram fazer algum tipo de avaliação da ansiedade. Nenhum participante relatou utilizar técnicas semiobjetivas do tipo questionário estruturado. Aproximadamente $32 \%$ dos profissionais relataram utilizar um protocolo específico para a abordagem do paciente ansioso, sendo os mais utilizados a conversa (38\%), a prescrição farmacológica (23\%) ou algum tipo de condicionamento do paciente $(17 \%)$. O trabalho discute a importância de incorporar no ensino da odontologia conhecimentos advindos da psicologia, favorecendo o desenvolvimento de habilidades relacionadas ao diagnóstico e o manejo da ansiedade odontológica.
\end{abstract}

Palavras-chave: psicologia; odontologia; ansiedade; tratamento odontológico; comportamento; diagnóstico de ansiedade.

\begin{abstract}
Diagnosis and Treatment of Dental Anxiety by Dentists

This work verifies the knowledge and use of psychometric scales and cognitive-behavioral techniques by dentists. Professionals completed a self-administered questionnaire on diagnosis and treatment of dental anxiety. The number of participants was 967 dentists of which approximately $65 \%$ reported making some type of anxiety assessment. No participant reported using semi-objective techniques of assessment. Approximately $32 \%$ of professionals reported using a specific protocol for addressing the anxious patient, the most utilized were conversation (38\%), drug prescription (23\%) or some kind of conditioning of the patient (17\%). The paper discusses the importance of incorporating the teaching of psychological knowledge on dental education, favoring the development of skills related to the diagnosis and management of dental anxiety.
\end{abstract}

Keywords: Psychology; dentistry; anxiety; dental anxiety; behavior; anxiety assessment.

$\mathrm{Na}$ área da odontologia, historicamente é possível distinguir três momentos na evolução da formação dos cirurgiões-dentistas. Num primeiro momento a formação do profissional foi voltada principalmente para os aspectos mecânicos do tratamento odontológico, em que o trabalho do cirurgião-dentista pode ser comparado ao de um artesão, que tem na sua habilidade artística e no manuseio dos instrumentos o foco da sua atividade. Observa-se então o surgimento de um se- gundo momento em que o foco é dirigido para o aspecto biológico do tratamento odontológico, envolvendo o estudo das ciências fisiológicas, morfológicas e dos aspectos microbiológicos envolvidos no tratamento das doenças bucais. Finalmente, o terceiro momento da educação odontológica pode ser chamado de psicossocial, pois passa a considerar que o dentista deve ser capaz de cuidar da pessoa como um todo (Dworkin, 2001). Nesta dimensão, postula-se que é

* Endereço para correspondência: Rodrigo Dutra Murrer - murrer@gmail.com 
preciso que o estudante seja capaz de enxergar além dos órgãos e sistemas de que o organismo é composto, mas o ser humano, que reage a um ambiente físico e social em contínuo processo de mudança. E é exatamente nesse momento que se criam as possibilidades reais de inserção das ciências comportamentais em Odontologia e certamente em outras áreas de saúde (Moraes, 1999).

A psicologia aplicada à odontologia pode ser definida como um sistema de conhecimentos teóricos e técnicos, derivados da psicologia clínica da saúde, aplicado às atividades de avaliação, controle e modificação de repertórios de comportamentos de indivíduos expostos a tratamento odontológico ou envolvidos em situações de cuidados de saúde bucal, incluindo familiares e cuidadores (Moraes et al., 2006). O objetivo principal da psicologia aplicada à odontologia é intervir nos aspectos psicossociais que estão presentes nos processos de diagnóstico, tratamento e reabilitação em odontologia, procurando promover e manter o estado geral de saúde do indivíduo, bem como prevenir e facilitar o enfrentamento eficiente de situações de tratamento dos transtornos bucais de usuários de sistemas de saúde. Por se tratar de uma campo interdisciplinar, requer a colaboração mútua e a integração de conhecimentos da psicologia, da odontologia e de outras ciências da saúde (Moraes \& Pessotti, 1985).

Reconhecidamente, muitas situações de tratamento de saúde apresentam um potencial ansiogênico, especialmente aquelas relacionadas a procedimentos médicos e odontológicos. Este campo de atuação e cuidado permite o desenvolvimento de pesquisas que investiguem aspectos relacionados às alterações psicofisiológicas do paciente e a possíveis estratégias que auxiliem o paciente a lidar com os aspectos adversos do tratamento (Giron, 1988). Compreender os fatores ansiogênicos que são despertados nas situações de dor, de doenças e de procedimentos utilizados na odontologia é necessário e fundamental para que aconteça uma conscientização geral de que o cirurgião-dentista não trata apenas de uma boca e sim de uma boca que faz parte de um ser humano que tem uma história, com passado, presente e futuro, e que está inserido na complexidade do contexto sócioeconômico-cultural em que vive (Duarte, 1989). Estas preocupações apresentam relevância não apenas científica, como também implicam em contribuições clínicas, educacionais e sociais do psicólogo e de outros profissionais da saúde que, por sua vez, mostraram-se um campo promissor para o desenvolvimento de estratégias cognitivo-comportamentais e para a discussão de fenômenos psicológicos e fisiológicos como a ansiedade (Hafner, 2011).

Apesar dos grandes avanços tecnológicos ocorridos na odontologia nas últimas décadas, a ansiedade em relação ao tratamento odontológico continua prevalente entre os pacientes, sendo apontada como um fenômeno universal, presente em países de culturas diversas espalhados pelo mundo (Kritsidima, Newton, \& Asimakopoulou, 2010; Lahmann et al., 2008; Ng \& Leung, 2008; Nicolas, Collado, Faulks, Bullier, \& Hennequin, 2007), inclusive no Brasil (Bottan, Oglio, \& Araujo, 2007; Cardoso \& Loureiro, 2005; Kanegane, Penha, Borsatti, \& Rocha, 2006).

Numa perspectiva analítico-comportamental a ansiedade pode ser definida como sendo um estado que abrange excitação biológica ou manifestações autonômicas e musculares (taquicardia, respostas galvânicas da pele, hiperventilação, sensações de afogamento ou sufocamento, sudorese, dores e tremores), redução na eficiência comportamental (decréscimo em habilidades sociais, dificuldade de concentração), respostas de fuga e/ou esquiva (o que sugere expectativa ou um controle por eventos futuros) e relatos verbais de estados internos desagradáveis (angústia, apreensão, medo, insegurança, mal-estar indefinido, etc.) (Zamignani \& Banaco, 2005). Sob este ponto de vista, a ansiedade é usualmente entendida como uma condição resultante da presença de um estímulo que precede um evento adverso, ou seja, é uma resposta diante de um provável evento aversivo e é considerada um padrão de responder do indivíduo que é aprendido em diversos contextos. Este processo pode ser descrito como uma resposta diante de um estímulo que precede uma situação aversiva. Na história de aprendizado de cada indivíduo, este é exposto a diversas experiências aversivas. Esta exposição resulta no aprendizado de respostas que terminam ou adiam eventos aversivos. Por exemplo, ter de se submeter a uma extração dental pode desencadear respostas de ansiedade e a pessoa pode adiar este evento (Zanatta, 2011).

A ansiedade ainda representa uma séria barreira à utilização dos serviços odontológicos, mesmo quando muito necessários, resultando em efeitos detrimentais à saúde bucal (Bottan et al., 2007; Coulson \& Buchanan, 2008; Heft, Meng, Bradley, \& Lang, 2007; Lahmann et al., 2008; Vermaire, de Jongh, \& Aartman, 
2008), podendo também levar a graves problemas de saúde como a pneumonia, infecção urinária, febre, septcemia, mediastinites, extensão intracranial de abcessos periapicais, osteomielites faciais, sinusites e sepsis (Mehrstedt, John, Tonnies, \& Micheelis, 2007). Além disso, autores relatam que indivíduos com ansiedade odontológica usam mais medicamentos, tem normalmente um maior consumo de álcool e drogas ilícitas e uma alta frequência de faltas no emprego devido a doenças, resultando em prejuízos em escala global (Berggren, 2001; Mehrstedt et al., 2007), estando associada também a problemas sociais e ocupacionais (Peltier, 2009).

Há evidências de que quando o cirurgião-dentista sabe do grau de ansiedade odontológica do paciente essa ansiedade se reduz, provavelmente porque o profissional muda sua abordagem, prestando mais atenção nos sentimentos do paciente e, além disso, o paciente se tranquiliza acreditando que o dentista, sabendo da sua condição ansiosa, irá tratá-lo de maneira mais favorável (Dailey, Humphris, \& Lennon, 2002). Portanto, um dos aspectos importantes no estudo da ansiedade é a sua mensuração e quantificação, de modo a permitir um correto diagnóstico e uma abordagem adequada por parte do cirurgião-dentista. É desejável portanto que para avaliar a ansiedade o profissional utilize um instrumento com propriedades psicométricas de validade e confiabilidade comprovadas (Tunc, Firat, Onur, \& Sar, 2005; Yuan, Freeman, Lahti, Lloyd-Williams, \& Humphris, 2008).

Atualmente, existem várias técnicas utilizadas para medir a ansiedade, sendo possível agrupá-las em seis grandes grupos: subjetivas (impressão clínica global baseada numa entrevista não estruturada), sistemáticas (abordam a ansiedade nas mais variadas vertentes utilizando questionários), focais (focam apenas um aspecto da ansiedade), não-objetivas (testes projetivos), semiobjetivas (utilizando questionários em que os sinais e sintomas específicos são apresentados bem como as instruções para os quantificar) e objetivas (utilizando variáveis psicofisiológicas, como cardiovasculares, respiratórias, músculo-esqueléticas) (Lopes, Ponciano, Pereira, Medeiros, \& Kleinknecht, 2004).

Uma vez diagnosticada a presença da ansiedade odontológica cabe então ao profissional selecionar o tipo de abordagem mais indicada para o manejo do paciente.
A intervenção psicológica paralela ao tratamento odontológico tem ajudado alguns pacientes a encarar procedimentos odontológicos considerados aversivos (injeção anestésica, utilização de motores, etc.), o que pode reduzir o nível de ansiedade geralmente manifestado por indivíduos com história de medo ou de não colaboração com tratamento bucal (Moraes et al., 2006)

Técnicas farmacológicas como a utilização de óxido nitroso, anti-histamínicos, tranquilizantes menores, sedativos não barbitúricos e até anestesia geral tem sido utilizadas (Aartman, 1998; Smyth, 1993; Woodmansey, 2005) e, apesar destes métodos melhorarem bastante algumas das condições dos pacientes, os medicamentos não resolvem todos os problemas associados a estas situações e normalmente possuem alguns efeitos colaterais (Montgomery, David, Winkel, Silverstein, \& Bovbjerg, 2002; Montgomery et al., 2010).

Por este motivo, as técnicas de psicologia comportamental, relaxamento e hipnoterapia tem assumido um lugar de destaque enquanto regimes de tratamento eficazes (Frenay, Faymonville, Devlieger, Albert, \& Vanderkelen, 2001; Gerschman, 1989; Woodmansey, 2005). Algumas pesquisas, com acompanhamento até de sete anos, têm mostrado que procedimentos da terapia comportamental podem reduzir efetivamente o medo e promover visitas regulares ao cirurgião-dentista de pacientes diagnosticados com alta ansiedade odontológica (Berggren, 1986, 2001; Berggren \& Linde, 1984). Outros trabalhos mostram que diferentes intervenções comportamentais conseguem bons resultados na diminuição da ansiedade (Berggren, Hakeberg, \& Carlsson, 2000; Bottan et al., 2007; Clark \& Hirschman, 1980).

Existe um rico corpo de dados científicos dando suporte para a utilização de estratégias comportamentais para diminuição da ansiedade e dor associados a procedimentos médicos, particularmente os invasivos (Cohen, 2008). Parte das técnicas utilizadas requerem exposição do indivíduo ao estímulo fóbico de maneira a produzir a extinção da reação exagerada de medo. É possível também usar tratamentos cognitivos, que tem como objetivos alterar e reestruturar o conteúdo de cognições negativas e aumentar o controle sobre os pensamentos negativos do paciente, visando assim modificar o comportamento por meio de abordagem dirigida para atenção, consciência, mediação, repertorio de resposta, componentes motivacionais e de in- 
centivo (Berggren, 2001; Kvale, Berggren, \& Milgrom, 2004).

Assim, considerando o impacto da ansiedade odontológica na população e a importância dos conhecimentos teóricos e técnicos, derivados da psicologia clínica da saúde, aplicado às atividades de avaliação, controle e modificação de repertórios de comportamentos de indivíduos expostos a tratamento odontológico, o objetivo deste trabalho foi verificar o conhecimento e a prática profissional relacionados às formas de diagnóstico e manejo da ansiedade odontológica pelos cirurgiões-dentistas.

\section{MÉTODO}

Foi solicitado a cirurgiões-dentistas dos municípios de Anápolis, Goiânia e Distrito Federal que preenchessem um questionário autoaplicável (Anexo 1) com perguntas referentes a idade, gênero, formação acadêmica, prática profissional, diagnóstico e tratamento da ansiedade odontológica. Houve a intenção de envolver o maior número possível de profissionais, de grandes municípios da região Centro Oeste de maneira a ter uma amostra que refletisse com a maior precisão possível a realidade regional. Optou-se por deixar que os profissionais respondessem de forma aberta às perguntas referentes a métodos de diagnósti- co e manejo da ansiedade para não restringir as possibilidades de resposta.

Os cirurgiões-dentistas foram procurados em seus ambientes de trabalho (consultórios e clínicas) e em locais e situações de concentração destes profissionais (congressos, jornadas, votações do Conselho Regional de Odontologia) e convidados a participar da pesquisa. Em caso de concordância, individualmente, de maneira reservada, neste mesmo local foi apresentado o Termo de Consentimento Livre e Esclarecido que uma vez assinado foi arquivado e em seguida apresentado o questionário para preenchimento. No total, 967 profissionais responderam o questionário que, depois de preenchidos, foram armazenados separados dos Termos de Consentimento e posteriormente processados, analisados por meio de estatística descritiva para a tabulação dos resultados.

\section{RESULTADOS}

No total foram 967 cirurgiões-dentistas preencheram o questionário, sendo 252 no município de Anápolis-GO, 521 em Goiânia-GO e 194 em Brasília-DF. $O$ perfil dos participantes em relação a gênero, idade e qualificação acadêmica está descrito nas tabelas abaixo:

Tabela 1

Distribuição dos Profissionais Participantes Segundo Gênero

Gênero

Feminino

Masculino

Tabela 2

Distribuição dos Profissionais Participantes Segundo Idade

\section{Faixa etária}

Porcentagem

21-30 anos

$31,22 \%$

31-40 anos

41-50 anos

51-60 anos

61-70 anos

$71-80$ anos

\section{Porcentagem}

$56,78 \%$

$43,22 \%$
$19,62 \%$

$9,07 \%$

$2,32 \%$

$0,32 \%$ 
Tabela 3

Distribuição dos Profissionais Participantes Segundo Qualificação Acadêmica

\begin{tabular}{lc} 
Titulação & Porcentagem \\
Graduação & $35,03 \%$ \\
Especialização & $57,99 \%$ \\
Mestrado & $5,50 \%$ \\
Doutorado & $1,48 \%$ \\
\hline
\end{tabular}

Dos profissionais participantes aproximadamente $65 \%$ relataram fazer algum tipo de avaliação da ansiedade do paciente, entre estes $15 \%$ não descreveram o que fazem e os outros $85 \%$ relataram utilizar técnicas subjetivas de avaliação (Figura 1). Nenhum dos pro- fissionais avaliados relatou utilizar técnicas semiobjetivas do tipo questionário estruturado ou qualquer outro tipo de escala psicométricas cujas propriedades tenham sido testadas quanto a validade e a confiabilidade.

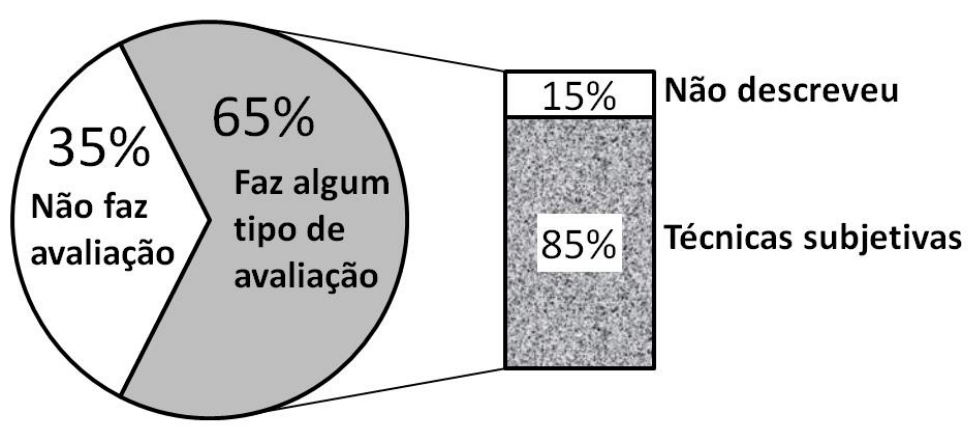

Figura 1. Distribuição dos profissionais segundo a utilização de procedimento para a avaliação da ansiedade do paciente.

Ainda a respeito do tema ansiedade odontológica, aproximadamente $32 \%$ dos profissionais relataram utilizar um protocolo específico para a abordagem do paciente ansioso. Entre estes somente $85 \%$ descreveram o protocolo utilizado: $38 \%$ utiliza a conversa, $23 \%$ utiliza prescrição farmacológica, $17 \%$ utiliza algum tipo de condicionamento do paciente, sendo que entre estes a grande maioria utiliza a dessensibilização sistemática, $17 \%$ utiliza alguma outra abordagem como encaminhamento para psicólogo ou médico e $5 \%$ utiliza algum tipo de técnica de relaxamento (Figura 2).

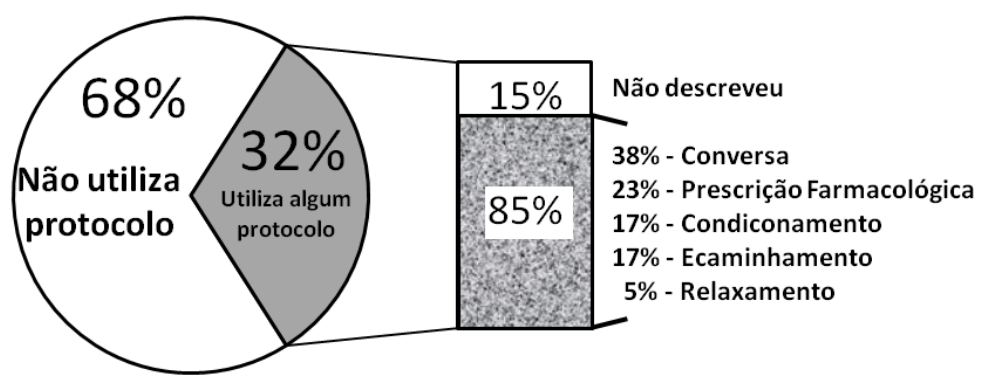

Figura 2. Distribuição dos profissionais segundo a utilização de protocolos de abordagem do paciente ansioso. 


\section{DISCUSSÃO}

Apesar dessa grande variedade de instrumentos com validade psicométrica, este trabalho verificou que $35 \%$ dos cirurgiões-dentistas participantes não fazem qualquer tipo de avaliação da ansiedade do paciente e que, entre os que fazem avaliação, a única forma de avaliação de ansiedade utilizada pelos profissionais foi a avaliação subjetiva. Esta constatação é preocupante pois, da mesma forma que para fazer o plano de tratamento o cirurgião-dentista leva em consideração vários fatores, é desejável que também fosse feita uma avaliação estruturada da ansiedade odontológica do paciente (Berggren, 2001; Dailey, Humphris, \& Lennon, 2001; Facco, Zanette, \& Manani, 2008).

Em de uma perspectiva de integração de conhecimentos da área da Psicologia na prática odontológica é primordial para o profissional que deseja fazer um bom trabalho conhecer minimamente os conteúdos da psicologia aplicada à odontologia e adquirir um conhecimento mais profundo do sentimento de medo e o quanto este pode afetar não só a relação profissionalpaciente, mas também a execução dos procedimentos no consultório (Ferreira, Manso, \& Gavinha, 2008). Além disso, alguns trabalhos demonstram que se o cirurgião-dentista tiver conhecimento prévio do nível de ansiedade dos pacientes, ele poderá tomar medidas mais adequadas para aliviar a ansiedade durante o tratamento (Corah, Gale, \& Illig, 1978; Yuan et al., 2008). Na verdade, toda a equipe odontológica precisa ter um cuidado tanto com crianças como com pacientes adultos e utilizar técnicas apropriadas de comunicação que aumentem a confiança e a sensação de controle dos mesmos, pois a abordagem preventiva diminui incidência de ansiedade odontológica em indivíduos psicologicamente vulneráveis (Locker, Liddell, Dempster, \& Shapiro, 1999).

Infelizmente, muitos profissionais desconhecem a existência de instrumentos específicos para este fim (Dailey et al., 2001), até porque a observação e a identificação de comportamentos e mudanças comportamentais não são tarefas fáceis para o cirurgiãodentista clínico, cuja formação raramente inclui treino observacional de comportamento ou de manejo em ciências do comportamento (Piko \& Kopp, 2004). Poucas faculdades oferecem treinamento sistemático em técnicas básicas de reconhecimento de pacientes ansiosos e fóbicos e de manejo de comportamento (Kaakko, Getz, \& Martin, 1999; Possobon, Carrascoza, Moraes, \& Costa Jr, 2007). Verifica-se que a atuação do dentista ainda tem um foco preponderantemente tecnicista, produto de uma sistemática de ensino que dá ênfase no ensino e reforçamento de habilidades manuais e procedimentos tecnológicos. Isso contrasta, muitas vezes, com a necessidade de uma atuação mais humanística, exigida pela própria situação de proximidade física e pelo estado emocional vulnerável de muitos pacientes. Observa-se que um profissional preocupado apenas com o procedimento a ser realizado pode não perceber manifestações de ansiedade e, por isso, não oferecer o amparo necessário e imediato ao paciente (Moraes, Sanchez, Possobon, \& Costa Júnior, 2004; Possobon et al., 2007).

Uma vez diagnosticado, o manejo do paciente ansioso deveria ser feito de maneira sistemática e baseado em evidências científicas. Verificou-se que apenas $32 \%$ dos profissionais participantes utiliza um protocolo específico para abordagem desses casos, dentre estes, 38\% utiliza apenas uma conversa não estruturada para "acalmar" o paciente. A segunda abordagem mais utilizada pelos profissionais participantes foi a prescrição farmacológica, utilizada por $23 \%$ deles, apesar de saber-se que os medicamentos não resolvem todos os problemas associados a estas situações e normalmente possuem alguns efeitos colaterais (Montgomery et al., 2002; Montgomery et al., 2010).

Verificou-se, portanto que apenas uma pequena parcela dos profissionais (17\%) utiliza algum tipo de condicionamento do paciente, sendo que entre estes a grande maioria declarou utilizar a dessensibilização sistemática ou algum tipo de técnica de relaxamento e $17 \%$ utiliza alguma outra abordagem como encaminhamento para psicólogo ou médico. Esse achado evidencia uma provável defasagem na aplicação pelos cirurgiões-dentistas das evidências científicas da área da Psicologia disponíveis sobre a eficácia de diversas formas de tratamento da ansiedade odontológica (Aartman, de Jongh, Makkes, \& Hoogstraten, 2000; Berggren, 2001; Berggren et al., 2000; Cohen, 2008; Frenay et al., 2001; Kleinhauz, Eli, Baht, \& Shamay, 1992; Lahmann et al., 2008; Moore, Brodsgaard, \& Abrahamsen, 2002; Smyth, 1993). Essa defasagem é provavelmente resultado da ênfase dada pelas faculdades de Odontologia e seus currículos na aquisição de habilidades técnicas e de precisão, sendo que só recentemente e por algumas escolas vem sendo reconhecido que os aspectos comportamentais e emocionais dos pacientes e dos dentistas são essenciais para o 
resultado final do tratamento odontológico (Eli \& Kleinhauz, 1992).

Há, portanto, necessidades e benefícios que justificam uma melhora na educação relacionada ao ensino da psicologia aplicada à odontologia. Um melhor conhecimento a respeito de métodos eficazes pode melhorar o tratamento odontológico para os pacientes com ansiedade odontológica e prevenir o agravamento dos medos (Berggren, 2001). A psicologia tornou-se um importante componente da educação e pesquisa odontológicas (Newton \& Buck, 2000) e é importante que as pessoas envolvidas com o ensino odontológico de graduação e pós-graduação sejam sensibilizadas para a necessidade de incluir na formação do cirurgião-dentista habilidades e competências relacionadas ao diagnóstico, à identificação e ao manejo da ansiedade odontológica (Bottan et al., 2007; Humphris, Dyer, \& Robinson, 2009; Possobon et al., 2007; Seger \& Ferreira, 1998; Wolf, 2002).

\section{CONSIDERAÇÕES FINAIS}

Dworkin (2001), considerando a necessidade de expandir o papel dos cirurgiões-dentistas para contribuir com a saúde e o bem-estar geral dos pacientes, propõe que o dentista seja considerado como um clínico biocomportamental, cujo objetivo é prevenir doenças, promover e manter a saúde geral e bucal e atuar para que as situações de tratamento odontológico sejam vivenciadas da melhor forma possível por todas as pessoas envolvidas.

Naturalmente essa mudança na forma de atuação passa necessariamente pela transformação da maneira como os profissionais são formados, o que implica alterações de paradigmas nos projetos pedagógicos dos cursos de graduação em odontologia.

Considerando-se os três momentos relacionados ao foco da prática profissional, os resultados deste trabalho permitem concluir que os cirurgiões-dentistas ainda não atingiram de fato o terceiro momento, de foco psicossocial. Tal constatação aponta para uma necessidade de se reforçar ainda mais a formação do cirurgião-dentista em termos dos conhecimentos da psicologia aplicada e de que os profissionais já formados procurem educar-se a respeito de métodos de diagnóstico mais aceitáveis e de técnicas de manejo do medo e da ansiedade que possam tornar a odontologia melhor para os pacientes e para a equipe odontológica.

\section{REFERÊNCIAS}

Aartman, I. H. (1998). Reliability and validity of the short version of the Dental Anxiety Inventory. Community Dentistry and Oral Epidemiology, 26(5), 350-354.

Aartman, I. H., de Jongh, A., Makkes, P. C., \& Hoogstraten, J. (2000). Dental anxiety reduction and dental attendance after treatment in a dental fear clinic: a follow-up study. Community Dentistry and Oral Epidemiology, 28(6), 435-442.

Berggren, U. (1986). Long-term effects of two different treatments for dental fear and avoidance. Journal of Dental Research, 65(6), 874-876.

Berggren, U. (2001). Long-term management of the fearful adult patient using behavior modification and other modalities. Journal of Dental Education, 65(12), 13571368.

Berggren, U., Hakeberg, M., \& Carlsson, S. G. (2000). Relaxation vs. cognitively oriented therapies for dental fear. Journal of Dental Research, 79(9), 1645-1651.

Berggren, U., \& Linde, A. (1984). Dental fear and avoidance: a comparison of two modes of treatment. Journal of Dental Research, 63(10), 1223-1227.

Bottan, E. R., Oglio, J. D., \& Araujo, S. M. d. (2007). Ansiedade ao tratamento odontológico em estudantes do ensino fundamental. Pesq Bras Odontoped Clin Integr, 7(3), 241-246.

Cardoso, C. L., \& Loureiro, S. R. (2005). Problemas comportamentais e stress em crianças com ansiedade frente ao tratamento odontológico. Estudos de Psico-logia (Campinas), 22, 5-12.

Clark, M., \& Hirschman, R. (1980). Effects of paced respiration on affective responses during dental stress. Journal of Dental Research, 59(9), 1533.

Cohen, L. L. (2008). Behavioral approaches to anxiety and pain management for pediatric venous access. Pediatrics, 122 Suppl 3, S134-139. DOI: 10.1542/peds.2008-1055f

Corah, N. L., Gale, E. N., \& Illig, S. J. (1978). Assessment of a dental anxiety scale. Journal of the American Dental Association, 97(5), 816-819.

Coulson, N. S., \& Buchanan, H. (2008). Self-reported efficacy of an online dental anxiety support group: a pilot study. Community Dentistry and Oral Epidemiology, 36(1), 43-46. DOI: 10.1111/j.1600-0528.2007.00349.x

Dailey, Y. M., Humphris, G. M., \& Lennon, M. A. (2001). The use of dental anxiety questionnaires: a survey of a group of UK dental practitioners. British Dental Journal, 190(8), 450-453. DOI: 10.1038/sj.bdj.4801000a

Dailey, Y. M., Humphris, G. M., \& Lennon, M. A. (2002). Reducing patients' state anxiety in general dental practice: a randomized controlled trial. Journal of Dental Re- 
search, 81(5), 319-322. DOI: 10.1177/1544059102 08100506

Duarte, I. (1989). Como tratar o medo de dentista? Psicologia: ciência e profissão, 9, 38-38.

Dworkin, S. F. (2001). The dentist as biobehavioral clinician. Journal of Dental Education, 65(12), 1417-1429.

Eli, I., \& Kleinhauz, M. (1992). Treatment of patients with oral related behavioural problems: a multidisciplinary approach. Dental World, 6, 10-11.

Facco, E., Zanette, G., \& Manani, G. (2008). Italian version of Corah's Dental Anxiety Scale: normative data in patients undergoing oral surgery and relationship with the ASA physical status classification. Anesthesia Progress, 55(4), 109-115. DOI: 10.2344/0003-3006-55.4. 109

Ferreira, M. A. F., Manso, M. C., \& Gavinha, S. (2008). Ansiedade e Fobia Dentária - Avaliação Psicométrica num Estudo Transversal. Revista Portuguesa de Estomatologia, Medicina Dentária e Cirurgia Maxilo-facial, 49(2), 77-86.

Frenay, M. C., Faymonville, M. E., Devlieger, S., Albert, A., \& Vanderkelen, A. (2001). Psychological approaches during dressing changes of burned patients: a prospective randomised study comparing hypnosis against stress reducing strategy. Burns, 27(8), 793-799. DOI: 10.1016/S0305-4179(01)00035-3

Gerschman, J. A. (1989). Hypnotizability and dental phobic disorders. Anesthesia Progress, 36(4-5), 131-137.

Giron, M. C. C. (1988). Fundamentos Psicológicos da Prática Odontológica. Porto Alegre: D. C. Luzzato Editores Ltda.

Hafner, M. B. (2011). Uso de Recurso Audio-Visual Informativo no Controle de Ansiedade e Dor em Pacientes Submetidos à Exodontia de Terceiro Molar. (Mestrado em Saúde da Criança e do Adolescente), UNICAMP, Campinas.

Heft, M. W., Meng, X., Bradley, M. M., \& Lang, P. J. (2007). Gender differences in reported dental fear and fear of dental pain. Community Dentistry and Oral Epidemiology, 35(6), 421-428. DOI: 10.1111/j.16000528.2006.00344.x

Humphris, G. M., Dyer, T. A., \& Robinson, P. G. (2009). The modified dental anxiety scale: UK general public population norms in 2008 with further psychometrics and effects of age. BMC Oral Health, 9, 20. doi: 10.1186/1472-6831-9-20

Kaakko, T., Getz, T., \& Martin, M. D. (1999). Dental anxiety among new patients attending a dental school emergency clinic. Journal of Dental Education, 63(10), 748752.

Kanegane, K., Penha, S. S., Borsatti, M. A., \& Rocha, R. G. (2006). Ansiedade ao tratamento odontológico no atendimento de rotina. $R G O, 54(2), 111-114$.
Kleinhauz, M., Eli, I., Baht, R., \& Shamay, D. (1992). Correlates of success and failure in behavior therapy for dental fear. Journal of Dental Research, 71(11), 18321835.

Kritsidima, M., Newton, T., \& Asimakopoulou, K. (2010). The effects of lavender scent on dental patient anxiety levels: a cluster randomised-controlled trial. Community Dentistry and Oral Epidemiology, 38(1), 83-87. DOI: 10.1111/j.1600-0528.2009.00511.x

Kvale, G., Berggren, U., \& Milgrom, P. (2004). Dental fear in adults: a meta-analysis of behavioral interventions. Community Dentistry and Oral Epidemiology, 32(4), 250-264. DOI: 10.1111/j.1600-0528.2004.00146.x

Lahmann, C., Schoen, R., Henningsen, P., Ronel, J., Muehlbacher, M., Loew, T., [...] Doering, S. (2008). Brief relaxation versus music distraction in the treatment of dental anxiety: a randomized controlled clinical trial. Journal of the American Dental Association, 139(3), 317324. DOI: 10.14219/jada.archive.2008.0161

Locker, D., Liddell, A., Dempster, L., \& Shapiro, D. (1999). Age of onset of dental anxiety. Journal of Dental Research, 78(3), 790-796.

Lopes, P. N., Ponciano, E., Pereira, A., Medeiros, J. A. M., \& Kleinknecht, R. A. (2004). Psicometria da Ansiedade Dentária: Avaliação das Características Psicométricas de uma Versão Portuguesa do Dental Fear Survey. Revista Portuguesa de Estomatologia, Medicina Dentária e Cirurgia Maxilofacial, 45(3), 133-145.

Mehrstedt, M., John, M. T., Tonnies, S., \& Micheelis, W. (2007). Oral health-related quality of life in patients with dental anxiety. Community Dentistry and Oral Epidemiology, 35(5), 357-363. DOI: $10.1111 / \mathrm{j} .1600-$ 0528.2007.00376.x

Montgomery, G. H., David, D., Winkel, G., Silverstein, J. H., \& Bovbjerg, D. H. (2002). The effectiveness of adjunctive hypnosis with surgical patients: a meta-analysis. Anesthesia and Analgesia, 94(6), 1639-1645, table of contents.

Montgomery, G. H., Hallquist, M. N., Schnur, J. B., David, D., Silverstein, J. H., \& Bovbjerg, D. H. (2010). Mediators of a brief hypnosis intervention to control side effects in breast surgery patients: response expectancies and emotional distress. Journal of Consulting and Clinical Psychology, 78(1), 80-88. DOI: 10.1037/a0017 392

Moore, R., Brodsgaard, I., \& Abrahamsen, R. (2002). A 3year comparison of dental anxiety treatment outcomes: hypnosis, group therapy and individual desensitization vs. no specialist treatment. European Journal of Oral Sciences, 110(4), 287-295.

Moraes, A. B. A. (1999). Psicologia e Saúde Bucal: circunscrevendo o campo. In R. R. Kerbauy (Ed.), Comportamento e Saúde: explorando alternativas. (pp. 61-83). Santo André: Editora ARBytes. 
Moraes, A. B. A., \& Pessotti, I. (1985). Psicologia aplicada à odontologia. São Paulo: Sarvier Editora.

Moraes, A. B. A., Possobon, R. F., Costa Jr., A. L., Fonseca, C. M. M., Silva, A. R. C., \& Rolim, G. S. (2006). Comportamentos não-colaborativos em Odontopediatria: a perspectiva da psicologia aplicada à odontologia. In: L. e. P. Crepaldi (Ed.), Temas em Psicologia Pediátrica. São Paulo: Casa do Psicólogo.

Moraes, A. B. A., Sanchez, K. A. S., Possobon, R. d. F., \& Costa Júnior, Á. L. (2004). Psicologia e odontopediatria: a contribuição da análise funcional do comportamento. Psicologia: Reflexão e Crítica, 17, 75-82.

Newton, J. T., \& Buck, D. J. (2000). Anxiety and pain measures in dentistry: a guide to their quality and application. Journal of the American Dental Association, 131(10), 1449-1457.

Ng, S. K., \& Leung, W. K. (2008). A community study on the relationship of dental anxiety with oral health status and oral health-related quality of life. Community Dentistry and Oral Epidemiology, 36(4), 347-356. DOI: 10.1111/j.1600-0528.2007.00412.x

Nicolas, E., Collado, V., Faulks, D., Bullier, B., \& Hennequin, M. (2007). A national cross-sectional survey of dental anxiety in the French adult population. BMC Oral Health, 7(12). DOI: 10.1186/1472-6831-7-12

Peltier, B. (2009). Psychological treatment of fearful and phobic special needs patients. Special Care in Dentistry, 29(1), 51-57. DOI: 10.1111/j.1754-4505.2008.00062.x

Piko, B. F., \& Kopp, M. S. (2004). Paradigm shifts in medical and dental education: behavioural sciences and behavioural medicine. European Journal of Dental Education, 8, Suppl 4, 25-31. DOI: 10.1111/j.1399-5863. 2004.00319.x

Possobon, R. d. F., Carrascoza, K. C., Moraes, A. B. A. d., \& Costa Jr, Á. L. (2007). O tratamento odontológico como gerador de ansiedade. Psicologia em Estudo, 12, 609-616.

Seger, L., \& Ferreira, M. C. D. (1998). Psicologia e odontologia: uma abordagem integradora: Santos.

Smyth, J. S. (1993). Some problems of dental treatment. Part 1. Patient anxiety: some correlates and sex differences. Australian Dental Journal, 38(5), 354-359.

Tunc, E. P., Firat, D., Onur, O. D., \& Sar, V. (2005). Reliability and validity of the Modified Dental Anxiety Scale (MDAS) in a Turkish population. Community Dentistry and Oral Epidemiology, 33(5), 357-362. DOI: 10.1111/j.1600-0528.2005.00229.x

Vermaire, J. H., de Jongh, A., \& Aartman, I. H. (2008). Dental anxiety and quality of life: the effect of dental treatment. Community Dentistry and Oral Epidemiology, 36(5), 409-416. DOI: 10.1111/j.1600-0528.2007.00416.x

Wolf, S. (2002). Psicologia no Consultório Odontológico. Editora Arte e Ciência.

Woodmansey, K. F. (2005). The prevalence of dental anxiety in patients of a university dental clinic. Journal of American College Health, 54(1), 59-61. DOI: 10.3200/JACH.54.1.59-61

Yuan, S., Freeman, R., Lahti, S., Lloyd-Williams, F., \& Humphris, G. (2008). Some psychometric properties of the Chinese version of the Modified Dental Anxiety Scale with cross validation. Health Qual Life Outcomes, 6(22). DOI: $10.1186 / 1477-7525-6-22$

Zamignani, D. R., \& Banaco, R. A. (2005). Um panorama analítico-comportamental sobre os transtornos de ansiedade. Revista Brasileira de Terapia Comporta-mental e Cognitiva, 7, 77-92.

Zanatta, J. (2011). Procedimento preparatório face a face e respostas de ansiedade e dor em jovens submetidos à exodontia de terceiro molar. (Tese de Mestrado em Saúde Coletiva), UNICAMP, Piracicaba. 
ANEXO 1 - Questionário de levantamento do conhecimento dos cirurgiões-dentistas a respeito do diagnóstico e manejo da ansiedade odontológica e da utilização da hipnose na odontologia.

\section{Questionário}

1- Qual a sua idade?

2- Gênero: ( ) feminino ( ) masculino

3- Marque abaixo sua qualificação acadêmica com o respectivo ano que você concluiu cada curso: (se tiver mais de uma especialização, colocar o ano daquela que terminou mais recentemente)

( ) Graduação - conclusão: ( ) Especialização - conclusão:

( ) Mestrado-conclusão: ( ) Doutorado - conclusão:

4- Você trabalha como clínico, atendendo pacientes? ( ) SIM ( ) NÃO

5- Você faz algum tipo de avaliação do medo e da ansiedade do paciente antes de atendê-lo? ( ) SIM ( ) NÃO Se você respondeu que sim: Qual avaliação você faz?

6- Você utiliza algum protocolo específico para lidar com pacientes que apresentam medo e ansiedade no consultório? () SIM () NÃO

Se você respondeu que sim: Qual é o protocolo utilizado? 